\title{
Energy Informatics
}

CrossMark

\author{
Bo Nørregaard Jørgensen
}

Correspondence: bnj@mmmi.sdu.dk University of Southern Denmark, Odense, Denmark

\section{Dear colleagues,}

Welcome to the inaugural issue of Energy Informatics, an international, peer-reviewed, open-source online journal. It is with a great sense of enthusiasm that we, on behalf of the editorial board, introduce this new journal. Energy informatics is a relatively young and profoundly interdisciplinary research field that has emerged over the past decades from the digitalization of the energy sector and its related industries.

As is the case for many new research fields, there is a noticeable absence of journals devoted solely to publishing research and technological advances in energy informatics. In lack of a primary outlet, researchers in energy informatics have to find the work of their peers scattered across many different journals covering topics related to energy, environment, buildings, transport, policy, etc.

We launched this journal because we believe the energy-informatics research community needs a journal that can cover the wide breadth and interdisciplinary nature of energy informatics. It is our ambition that Energy Informatics will become the primary journal for the publication and free-access to the most recent theoretical results and technological advances in the energy informatics field. To achieve this ambition, we are supported by an excellent editorial board consisting of more than 40 experts who cover the full breadth of energy informatics.

Since energy informatics is a young and very dynamic research field, we decided to support various types of article formats to facilitate and speed up the dissemination of new discoveries and insights in the field. The selected article formats provide authors with multiple options for presenting their work, whether it is theoretical, empirical, or policy-related. The following types of articles are accepted:

- Research articles reporting on original research results.

- Methodology articles presenting new experimental or computational methods, tests or procedures.

- Software articles describing tools likely to be of broad utility and which represent a significant advance over previously published software.

- Open dataset articles describing datasets that are made available for public use.

- Review articles providing systematic and substantial syntheses of specific research areas, evaluations of progress in specified areas, critical assessments with respect to issues, within the scope of Energy Informatics.

- Commentary, is a short, focused and opinionated article, stating the authors' position on any subject within the journal's scope. 
- Short communication, is an article reporting on new ideas, early observations and new findings, or discuss previously published articles in Energy Informatics or other journals.

- Short report is a presentation of research that extends previously published research, including the reporting of additional data and new findings.

- Project report, is an article describing a new research project or reporting on the achievements of a concluded research project.

- Meeting report, is an article describing the key developments presented and discussed at a meeting, as for instance IEA Annex meetings.

By supporting a broad range of article types, we hope to stimulate international dialog among academics, industry, retailers, investors, and policymakers with mutual interests in the energy sector.

Energy Informatics publishes original articles that address scientific, engineering, or societal topics related to the digitalization of the energy sector. Topics suitable for Energy Informatics include, but are not limited to;

- Innovation in data-driven energy services and business models

- Big Data-driven smart Energy Management Systems

- Management of data and information flow in the Digital Energy System 4.0

- Data analytics for energy-cost efficient system operation

- Privacy issues in energy data management

- Digitalization of energy production, delivery and consumption, including policy and strategy

- Digital technologies for enabling energy-aware user behavior

- Contextual computing for supporting human and energy system interactions

- Energy-efficient Cloud computing and data centers

- Cybersecurity issues for safe and reliable smart grid operation

- Social media's role in the transition towards sustainable energy

- Application of artificial intelligence and agent-based technology in smart energy systems

- Model-based and data-driven energy forecasting, including energy production and use

- Management of public and private procurement of digital energy technology

- Socioeconomic opportunities and barriers for adoption of digital energy technologies

- Sociotechnical aspects related to design and implementation of smart energy systems

- Innovation management in smart energy business eco-systems

- Digital entrepreneurship in emerging energy markets

- Smart grid communication architectures and protocols for improving grid resiliency

- Demand-side management, including demand response, dynamic pricing and incentive design

- Microgrid and distributed generation management and control

- Monitoring and control of Smart Buildings, including smart grid interoperability

In this inaugural issue of Energy Informatics, we are delighted to present five articles that together reflect the scientific, engineering, and societal scope of the journal. It was very encouraging to receive such diverse articles, as it shows the need for a journal dedicated to energy informatics. We would therefore like to thank our contributing authors. 
We begin this issue with an article written by Konstantin Hopf titled, "Mining Volunteered Geographic Information for Predictive Energy Data Analytics". The author presents a comprehensive review of crowd-sourced Volunteered Geographic Information (VGI) data sources relevant to energy data analysis, and demonstrates how two publicly available VGI data sources can help energy retailers and energy service providers to predict detailed information about their customers. The article's results highlight the value of VGI data sources for providing better customer service and its potential for targeting marketing campaigns based on predicted customer characteristics (Hopf 2018).

The second article, "Comparing Solar Photovoltaic and Battery Adoption in Ontario and Germany: An Agent-Based Approach", co-authored by Adedamola Adepetu, Ammar Alyousef, Srinivasan Keshav, and Hermann deMeer, uses Agent-Based Models to investigate the correlation between policy driven incentives and customer's adoption behavior of integrated photovoltaic and battery systems. The study highlights and explains differences between the two jurisdictions; Ontario in North America and Germany in Europe (Adepetu et al. 2018).

The third article, "Application of an Optimization-based Curtailment Service Provider Model in Real-Time Simulation", co-authored by Omid Abrishambaf, Pedro Faria, and Zita Vale, presents a study considering curtailment service providers as aggregators for small and medium-scale prosumers, who do not have adequate capacity to participate directly in the wholesale electricity markets. The authors investigate the feasibility of curtailment service providers as aggregators, using a novel real-time simulation model of a curtailment service provider in an experimental setting containing a distribution network with 20 consumers and prosumers, and 26 renewable-based producers including wind and photovoltaic generation. The authors provide interesting insights on the use of hardware-in-the-loop real-time simulations to understand how curtailment service providers and diverse mix of small and medium-scale prosumers can be integrated with the electricity market (Abrishambaf et al. 2018).

The fourth article, "Load Balancing of Renewable Energy: A Cyber Security Analysis", co-authored by Alexandre Louis Vernotte, Margus Välja, Matus Korman, Gunnar Björkman, Mathias Ekstedt, and Robert Lagerström, presents a cybersecurity analysis focusing on the ICT-part for load balancing of renewable energy sources in the smart grid. The analysis is based on an innovative load balancing centered smart grid reference architecture model that allows cyber-attacks and countermeasures to be introduced and analyzed. Based on their findings the authors raise concerns whether the architecture of the smart grid is satisfactory to meet the present and future cyber threats (Vernotte et al. 2018).

Finally, we conclude this first issue with an exciting project report, co-authored by Qianchuan Zhao, Li Xia, and Ziyan Jiang, presenting a newly launched smart building project in China. The article presents the project background, aims, partners, and key features of this project. The article provides valuable insight in the smart building research in China (Zhao et al. 2018).

Energy Informatics is intended to be a journal that serves, in a clear and optimal way, the publishing and reading needs of the energy informatics community. We hope that you enjoyed this first issue of Energy Informatics and that you will join us in our effort to make Energy Informatics a community journal by contributing to future issues of the journal. 
Author's contributions

The author read and approved the final manuscript.

\section{Competing interests}

The author declares that he has no competing interests

\section{Publisher's Note}

Springer Nature remains neutral with regard to jurisdictional claims in published maps and institutional affiliations.

Received: 11 July 2018 Accepted: 11 July 2018

Published online: 26 July 2018

References

Abrishambaf O, Faria P, Vale Z (2018) Application of an optimization-based curtailment service provider in real-time simulation. Energy Inform. https://doi.org/10.1186/s42162-018-0006-6

Adepetu A, Alyousef A, Keshav S, de Meer H (2018) Comparing Solar Photovoltaic and battery adoption in Ontario and Germany: an agent-based approach. Energy Inform. https://doi.org/10.1186/s42162-018-0012-8

Hopf K (2018) Mining volunteered geographic information for predictive energy data analytics. Energy Inform. https:// doi.org/10.1186/s42162-018-0009-3

Vernotte A, Välja M, Korman M, Björkman G, Ekstedt M, Lagerström R (2018) Load balancing of renewable energy: a cyber security analysis. Energy Inform. https://doi.org/10.1186/s42162-018-0010-x

Zhao Q, Xia L, Jiang Z (2018) Project report: new generation intelligent building platform techniques. Energy Inform. https://doi.org/10.1007/s42162-018-0011-9

Submit your manuscript to a SpringerOpen ${ }^{\circ}$ journal and benefit from:

- Convenient online submission

- Rigorous peer review

- Open access: articles freely available online

- High visibility within the field

- Retaining the copyright to your article

Submit your next manuscript at $>$ springeropen.com 